\title{
Outstanding reviewer award 2017
}

The editors and publisher of OR Spectrum are pleased to announce the following reviewers who have done an outstanding job in submitting reviews during the time of origin of volume 39 :

Marco Bijvank,

University of Calgary,

Calgary,

Canada

Murat Firat,

Eindhoven University of

Technology,

Eindhoven, Netherlands
Sophie Parragh,

Johannes Kepler Universität

Linz, Linz, Austria
Gudrun Kiesmüller,

Otto von Guericke

Universität Magdeburg, Magdeburg, Germany 